\title{
Hepatite fulminante: estudo dos fatores associados à mortalidade hos- pitalar de 100 pacientes priorizados para transplante de fígado
}

\section{Fulminant hepatic failure: factors associated with in hospital mortal- ity in hundred patients prioritized for liver transplantation}

\author{
Adriana Rochetto Assalin', Estela Regina Ramos Figueira ${ }^{2}$, \\ Joel Avancini Rocha Filho ${ }^{3}$, Telesforo Bacchella², Rodrigo C. T. Surjan², \\ Eleazar Chaib' ${ }^{2}$ Luiz Augusto Carneiro D'Albuquerque ${ }^{2}$
}

Assalin AR, Figueira ERR, Rocha Filho JA, Bacchella T, Surjan RCT, Chaib E, D'Albuquerque LAC. Hepatite fulminante: estudo dos fatores associados à mortalidade hospitalar de 100 pacientes priorizados para transplante de fígado. / Fulminant hepatic failure: factors associated with in hospital mortality in hundred patients prioritized for liver transplantation. Rev Med (São Paulo). 2013 jan.mar.;92(1):62-4.

RESUMO: Introdução. A despeito dos avanços nos cuidados de terapia intensiva e no transplante de fígado $(\mathrm{TF})$, a hepatite fulminante (HF) ainda hoje apresenta alta taxa de mortalidade. A identificação de fatores prognósticos de maior acurácia deve ajudar a otimizar a priorização dos pacientes em lista de espera para o TF. Objetivo. Avaliar fatores prognósticos de mortalidade hospitalar dos pacientes com HF priorizados para TF. Métodos. Foram estudados retrospectivamente 100 pacientes adultos (78 mulheres, idade média $35,5 \pm 14,7$ anos) com HF priorizados para TF, em um único centro, de fevereiro de 2002 a junho de 2011. O diagnóstico etiológico foi hepatite viral em $17 \%$ dos casos, medicamentosa em $29 \%$, autoimune em $13 \%$, criptogênica em $34 \%$ e outras causas em $7 \%$. A indicação do TF foi determinada de acordo com os critérios de O'Grady. Foram avaliados: idade, sexo, etiologia, intervalo icterícia/encefalopatia, intervalo entre a priorização e o TF, grau de encefalopatia, tempo de internação, RNI, fator V, bilirrubina, creatinina, AST, ALT, lactato e Model for End-Stage Liver Disease (MELD). Todos os dados foram coletados do dia da priorização. Resultados. O intervalo entre a priorização e o TF foi de 1,5 dias ( 0 a 9) e o tempo de internação foi de $18 \pm 27$ dias. A mortalidade hospitalar foi de $69 \%$. Os pacientes não sobreviventes apresentaram na priorização maior grau de encefalopatia [3 (1 a 4) vs. 2 ( 1 a 4$)$ ], MELD (41 \pm 9 vs. $38 \pm 7)$ e lactato $(62,2 \pm 45,2$ vs. $33,9 \pm 16,0 \mathrm{mg} / \mathrm{dL})$ quando comparados com os sobreviventes $(\mathrm{p}<0,05)$. Dos 100 pacientes, $69 \%$ foram submetidos ao TF, os outros $31 \%$ morreram antes do TF. Os pacientes não transplantados apresentaram maior grau de encefalopatia [4 (1 a 4) vs. 3 ( 1 a 4)], MELD ( $44 \pm 8$ vs. 38 $\pm 8)$, lactato $(78,4 \pm 48,3$ vs. $41,8 \pm 30,6 \mathrm{mg} / \mathrm{dL})$ e creatinina $(2,60 \pm 2,34$ vs. $1,55 \pm 1,54 \mathrm{mg} / \mathrm{dL})$ quando comparados aos pacientes submetidos ao TF $(\mathrm{p}<0.05)$. Conclusão. No momento da priorização para o TF, os pacientes com HF que apresentam condição clínica mais grave, com encefalopatia graus 3 ou 4, insuficiência renal, escores mais elevados de MELD e lactato elevado, têm maior taxa de mortalidade hospitalar mesmo quando submetidos ao TF, indicando pior prognóstico.

DESCRITORES: Hepatite/mortalidade; Transplante de fígado; Mortalidade hospitalar.

$2^{\circ}$ lugar Prêmio Painel Área Cirúrgica - XXXI Congresso Médico Universitário da FMUSP - COMU 2012.

${ }^{1}$ Disciplina de Pesquisa Científica em Medicina - FAPESP 2011/22470-3.

2. Disciplina de Transplante de Órgãos do Aparelho Digestivo - Departamento de Gastroenterologia da Faculdade de Medicina da Universidade de São Paulo. e-mail: estelafigueira@uol.com.br, eleazarchaib@yahoo.co.uk

3. Disciplina de Anestesiologia, Departamento de Cirurgia da Faculdade de Medicina da Universidade de São Paulo.

Endereço para correspondência: Departamento de Gastroenterologia. Disciplina de Transplante de Órgãos do Aparelho Digestivo FMUSP. Av. Dr. Enéas de Carvalho Aguiar, 255 - 9ª andar. CEP: 05403-000 - São Paulo, SP. 


\section{INTRODUÇÃO E OBJETIVO}

A despeito dos avanços nos cuidados de terapia intensiva e no transplante de fígado (TF), a hepatite fulminante (HF) ainda hoje apresenta alta taxa de mortalidade. A identificação de fatores prognósticos de maior acurácia deve ajudar a otimizar a priorização dos pacientes em lista de espera para o TF.

O objetivo do estudo foi avaliar fatores prognósticos de mortalidade hospitalar dos pacientes com HF priorizados para TF.

\section{MÉTODOS}

Foram estudados retrospectivamente 100 pacientes adultos com HF priorizados para TF, em um único centro, de fevereiro de 2002 a junho de 2011. A indicação do TF foi determinada de acordo com os critérios de O'Grady.

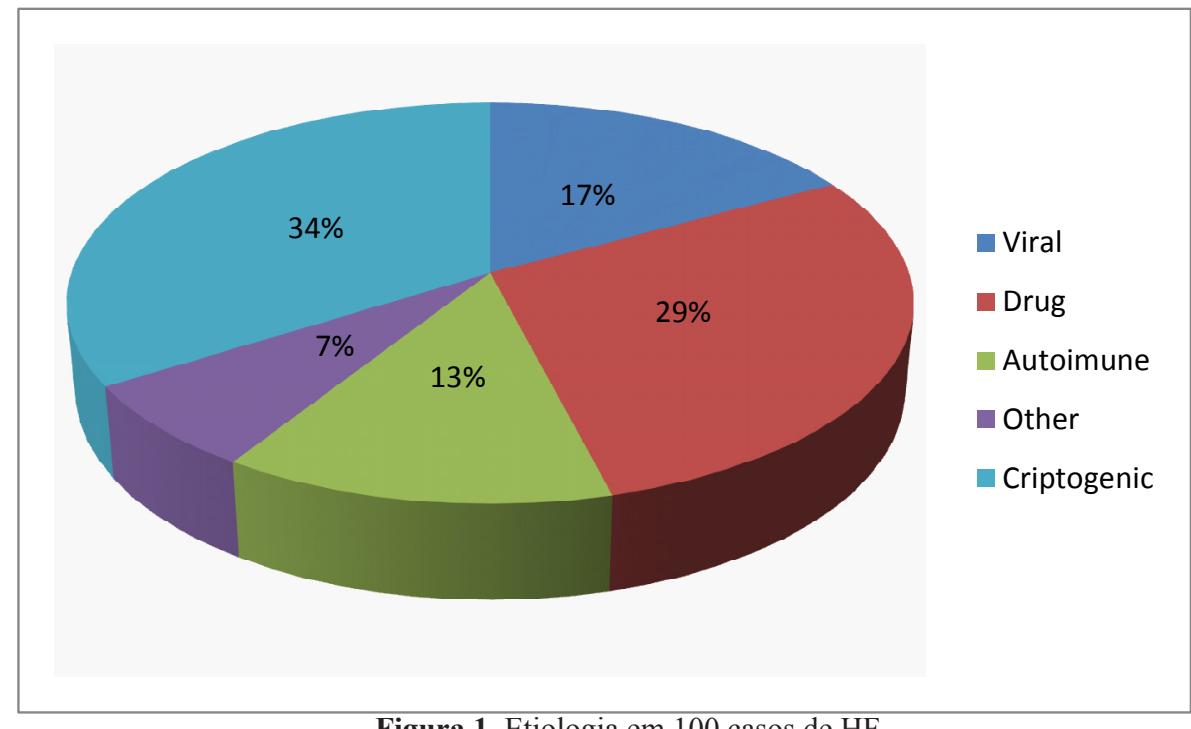

Figura 1. Etiologia em 100 casos de HF

Foram avaliados: idade, sexo, etiologia, intervalo icterícia/encefalopatia, intervalo entre a priorização e o TF, grau de encefalopatia, tempo de internação, RNI, fator V, bilirrubina, creatinina, AST, ALT, lactato e Model for End-Stage Liver Disease (MELD). Todos os dados foram coletados do dia da priorização.

\section{RESULTADOS}

Setenta e oito (78\%) pacientes eram mulheres e a idade média era 35,5 $\pm 14,7$ anos. O diagnóstico etiológico foi hepatite viral em $17 \%$ dos casos, medicamentosa em $29 \%$, autoimune em $13 \%$, criptogênica em $34 \%$ e outras causas em 7\%. O intervalo entre a priorização e o TF foi de 1,5 dias ( 0 a 9) e o tempo de internação foi de $18 \pm 27$ dias.

Os pacientes não sobreviventes apresentaram na priorização maior grau de encefalopatia [3 (1 a 4) vs. 2 (1 a 4)], $\operatorname{MELD}(41 \pm 9$ vs. $38 \pm 7)$ e lactato (62,2 $\pm 45,2$ vs. 33,9 $\pm 16,0 \mathrm{mg} / \mathrm{dL}$ ) quando comparados com os sobreviventes $(\mathrm{p}<0,05)$. Dos 100 pacientes, $69 \%$ foram submetidos ao $\mathrm{TF}$, os outros $31 \%$ morreram antes do TF.

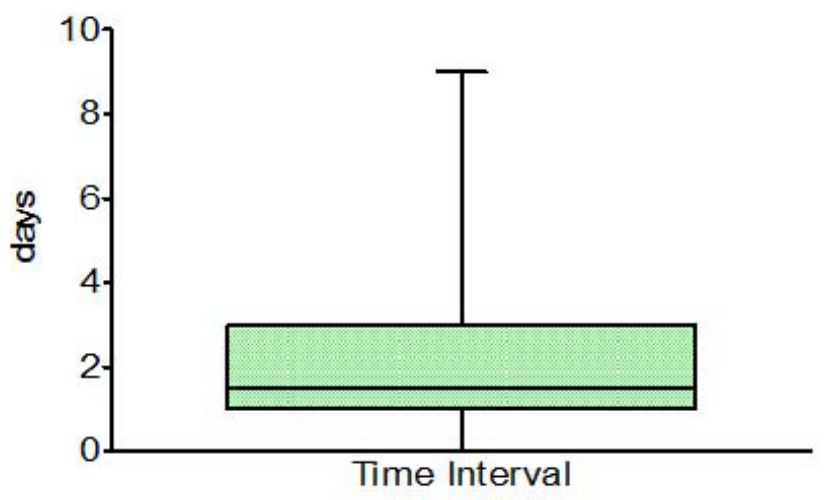

Figura 2. Intervalo entre a priorização e o TF ou a morte sem TF 
Os pacientes não transplantados apresentaram maior grau de encefalopatia [4 (1 a 4) vs. 3 (1 a 4)], MELD (44 \pm 8 vs. $38 \pm 8)$, lactato $(78,4 \pm 48,3$ vs. $41,8 \pm 30,6 \mathrm{mg} / \mathrm{dL})$

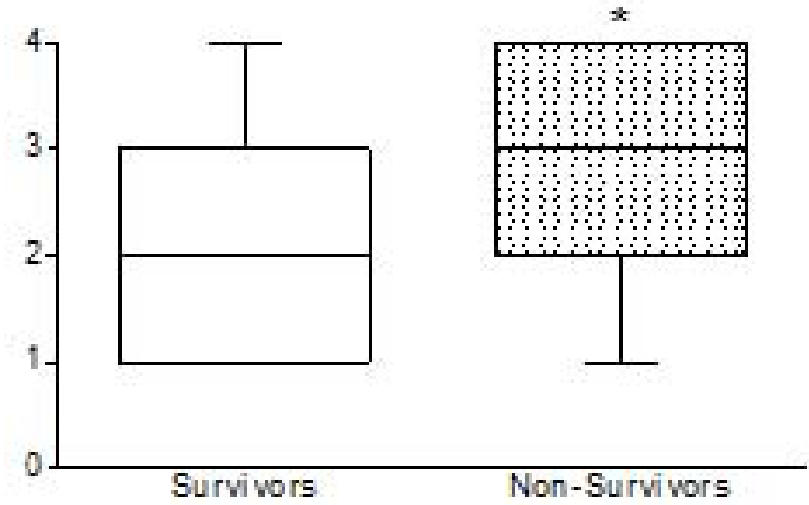

Figura 3. Grau de encefalopatia na priorização

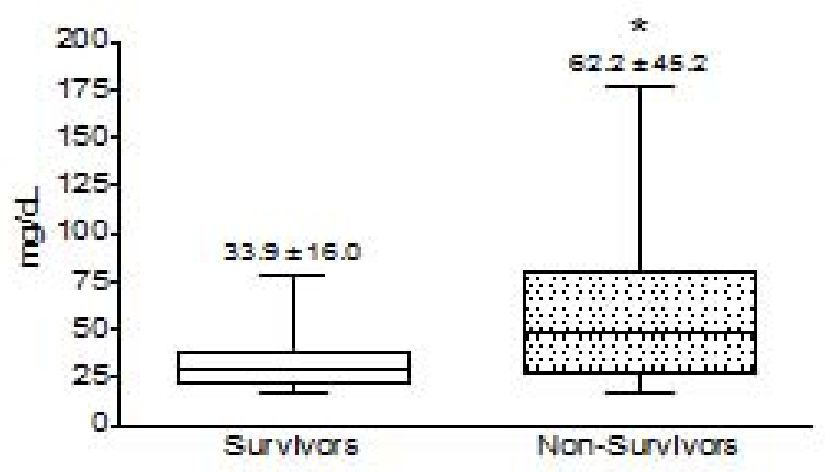

Figura 5. Lactato arterial na priorização

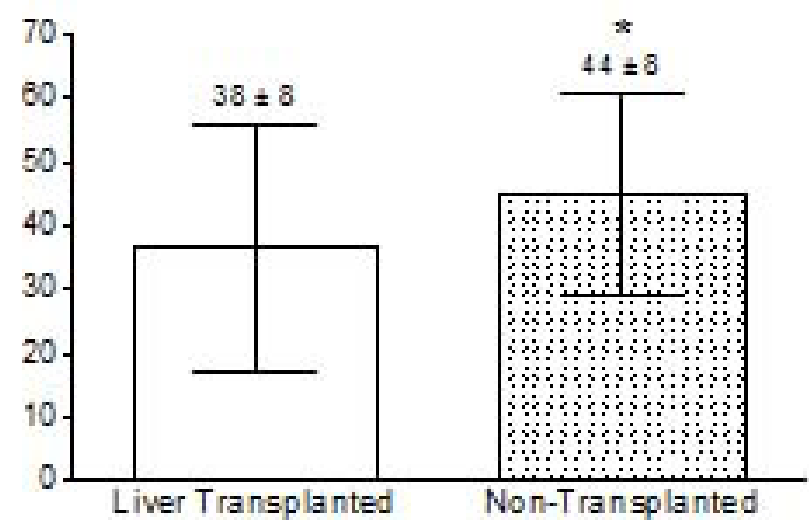

Figura 6A. Lactato arterial na priorização: comparação entre pacientes transplantados e não transplantados

\section{CONCLUSÃO}

No momento da priorização para o $\mathrm{TF}$, os pacientes com HF que apresentam condição e creatinina $(2,60 \pm 2,34$ vs. $1,55 \pm 1,54 \mathrm{mg} / \mathrm{dL})$ quando comparados aos pacientes submetidos ao TF $(\mathrm{p}<0.05)$.

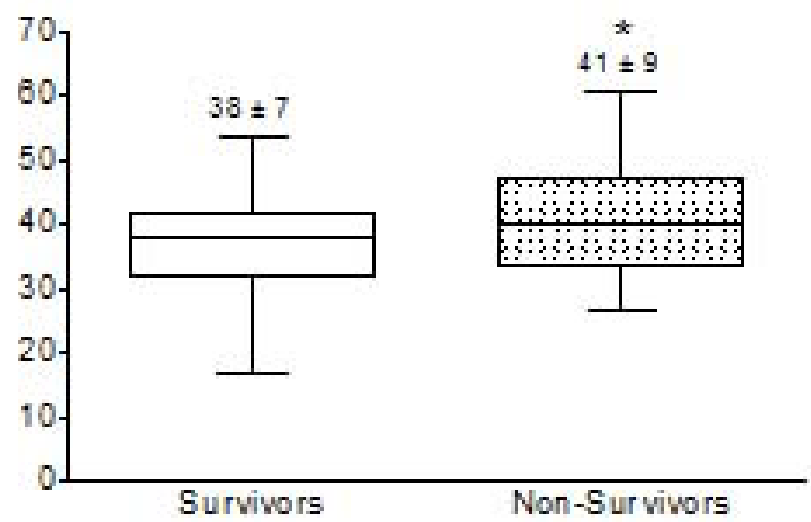

Figura 4. MELD na priorização

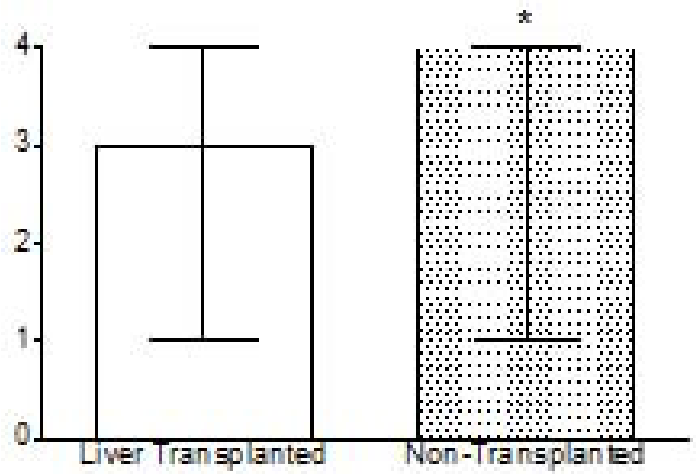

Figura 6. Grau de encefalopatia: comparação entre pacientes transplantados e não transplantados

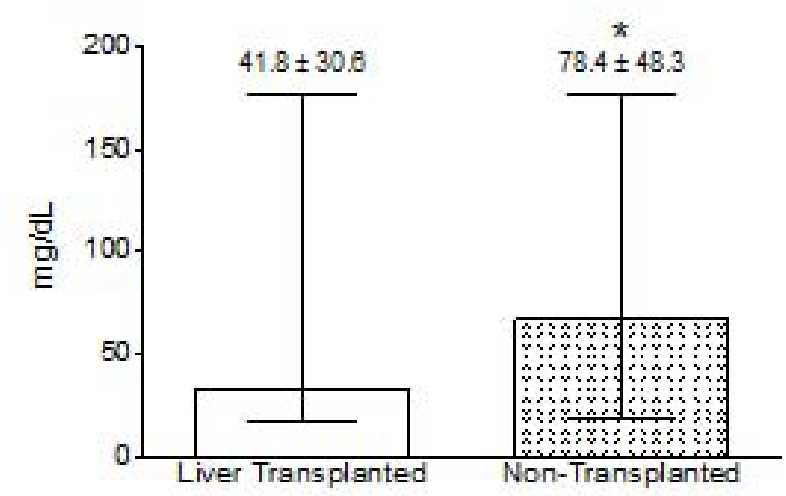

Figura 7. MELD: comparação entre pacientes transplantados e não transplantados

clínica mais grave, com encefalopatia graus 3 ou 4, insuficiência renal, escores mais elevados de MELD e lactato elevado, têm maior taxa de mortalidade hospitalar mesmo quando submetidos ao $\mathrm{TF}$, indicando pior prognóstico. 